\title{
Warsaw Variability Surveys
}

\author{
Janusz Kaluzny \\ Copernicus Astronomical Center, ul. Bartycka 18, 00-716 Warsaw, \\ Poland
}

\begin{abstract}
Four large-to-medium size photometric surveys are being conducted by Warsaw astronomers: the Warsaw-LCO survey of globular clusters, OGLE, DIRECT, and ASAS. In this contribution I provide short descriptions of these projects and discuss briefly some results obtained for pulsating variables.
\end{abstract}

\section{The Warsaw-LCO Survey of Globular Clusters}

The main goal of this survey is the identification of detached eclipsing binaries in globular clusters. Such binaries can be used as excellent distance and age indicators (Paczyński 1997). Thirteen globular cluster have been surveyed since 1993. Starting in 1997, most of the data are collected with the Las Campanas $1.0-\mathrm{m}$ Swope telescope equipped with a SITe $2048 \times 3150$ CCD giving a field of view $14.4 \times 22.8 \mathrm{arcmin}^{2}$. Some additional follow-up observations are conducted on the 2.5-m du Pont telescope. For a given cluster the total length of monitoring ranges from 30 to $150 \mathrm{hr}$, with the median value around $70 \mathrm{hr}$.

Precise and well-sampled light curves of RR Lyr and SX Phe stars are collected as a byproduct of the survey. These data become available through the Internet (http://www.camk.edu.pl/ jka) immediately after results for a given object are published. A list of monitored clusters and the numbers of variables identified in them are given in Table 1 . While monitoring M55 we identified five variables as being likely members of the Sagittarius dwarf galaxy: two RRab stars and three SX Phe variables.

\subsection{Nonradial Pulsators in M55}

Our sample of pulsating variables from the globular cluster M55 includes five newly identified RRc stars. The light curves of three of these stars exhibit changes in amplitude of over $0.1 \mathrm{mag}$ on the time scale shorter than a week. Detailed analyses indicate that the observed changes are most probably due to nonradial pulsations (Olech et al. 1999a).

At least 12 out of $24 \mathrm{SX}$ Phe variables identified in M55 show a presence of two or more periodicities in their light curves. Table 2 lists principal periods, period ratio $P_{1} / P_{2}$ and amplitudes for the first terms of Fourier series calculated for both periods after appropriate pre-whitening was applied. The derived values of $P_{1} / P_{2}$ indicate nonradial pulsations. In Fig. 1 we present light curves obtained on two consecutive nights for one of the multi-periodic SX Phe stars from M55. 
Table 1. Pulsating variables detected in the monitored GCs

\begin{tabular}{lrrl}
\hline Cluster & RR Lyr & SX Phe & \multicolumn{1}{c}{ Reference } \\
\hline$\omega$ Cen & 132 & 34 & Kaluzny et al. (1997a, 1997b) \\
NGC 288 & 3 & 5 & Kaluzny et al. (1997b) \\
NGC 4372 & 0 & 8 & Kaluzny et al. (1993) \\
NGC 6397 & 1 & 2 & Kaluzny (1997) \\
NGC 6362 & 18 & 4 & Mazur et al. (1999) \\
& 35 & 4 & Kaluzny et al., in prep \\
NGC 6752 & 0 & 3 & Thompson et al. (1999) \\
M3 & 42 & 1 & Kaluzny et al. (1998a) \\
M4 & 31 & 0 & Kaluzny et al., in prep. \\
M5 & 99 & 5 & Kaluzny et al. (1999b, 1999c) \\
M10 & 1 & 3 & Olech et al. (1999b) \\
M55 & 13 & & Olech et al. (1999a) \\
& & 24 & Kaluzny et al., in prep. \\
Ru 106 & 12 & 3 & Kaluzny et al. (1995a) \\
\hline Total & 387 & 96 & \\
\hline
\end{tabular}

We also note that the light curve of an SX Phe variable identified in M3 by Kaluzny et al. (1998) exhibits evidence for nonradial pulsations of that star.

\subsection{M3/M55 dichotomy}

Our sample of monitored clusters includes M3 and M55. These objects have similar metallicities and exhibit similar morphology of their color-magnitude diagrams (in particular their horizontal branches are similar). Both clusters are rich in blue stragglers, yet show very different relative frequencies of SX Phe
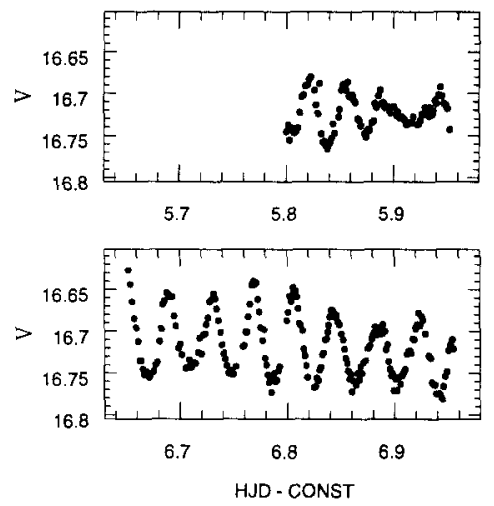

Figure 1. Light curve of one of the multi-periodic SX Phe stars in M55. 
Table 2. Multi-modal SX Phe stars in M55

\begin{tabular}{ccccc}
\hline ID & $P_{1}[\mathrm{~d}]$ & $P_{1} / P_{2}$ & $A_{1}^{1}$ & $A_{1}^{2}$ \\
\hline 19336 & 0.0388 & 1.017 & 0.020 & 0.009 \\
19407 & 0.0415 & 1.023 & 0.048 & 0.019 \\
25078 & 0.0593 & 1.034 & 0.027 & 0.018 \\
25204 & 0.0370 & 1.026 & 0.014 & 0.006 \\
31780 & 0.0487 & 1.075 & 0.035 & 0.010 \\
34347 & 0.0394 & 1.054 & 0.033 & 0.012 \\
34461 & 0.0438 & 1.023 & 0.025 & 0.011 \\
19084 & 0.0382 & 0.974 & 0.029 & 0.012 \\
12618 & 0.0358 & 1.049 & 0.017 & 0.008 \\
15539 & 0.0370 & 1.067 & 0.014 & 0.007 \\
19176 & 0.0452 & 1.239 & 0.053 & 0.024 \\
19480 & 0.0367 & 1.029 & 0.026 & 0.017 \\
\hline
\end{tabular}

stars. This can be illustrated by comparing numbers of blue stragglers for which we obtained good quality light curves with numbers of identified SX Phe stars:

$$
\begin{array}{ll}
\text { M55: } & N_{\mathrm{BS}}=40, N_{\mathrm{SX}}=24,[\mathrm{Fe} / \mathrm{H}]=-1.81 \\
\text { M3: } & N_{\mathrm{BS}}=25, N_{\mathrm{SX}}=1,[\mathrm{Fe} / \mathrm{H}]=-1.57
\end{array}
$$

Our data for M3 show several blue stragglers located inside the instability strip which do not show any variability exceeding about $0.02 \mathrm{mag}$ in the $V$ band.

\subsection{How Complete is the Sample of SX Phe Stars Identified in GCs?}

Figure 2 shows a full-amplitude versus period diagram for 96 SX Phe stars identified by our group in globular clusters. 53 of these stars exhibit light curves with full ranges not exceeding $0.10 \mathrm{mag}$ in the $V$ band. Many variables show amplitudes approaching the detection limit of our survey which, for most clusters, reaches $0.02-0.03 \mathrm{mag}$. That indicates that a significant fraction of SX Phe stars residing in the surveyed cluster was most likely missed.

\section{The OGLE-1 Survey}

The OGLE project (http://sirius.astrouw.edu.pl/ ftp/ogle/; Udalski et al. 1993) is aimed primarily at detection and photometry of microlensing events in the Galactic bulge, LMC, and SMC. The first phase of the survey, referred to here as OGLE-1, was conducted at Las Campanas Observatory on the 1.0-m Swope telescope during four seasons covering the period 1992-1995. About $2 \times 10^{6}$ stars in $2015 \times 15$ arcmin $^{2}$ fields toward the Galactic bulge were monitored. Twenty microlensing events were detected (Wozniak \& Szymanski 1998). Five parts of the Variable Star Catalog were published, containing 2861 stars from the Galactic bulge (Udalski et al. 1997b). That catalog includes 269 pulsating objects, mostly RR Lyr stars. 


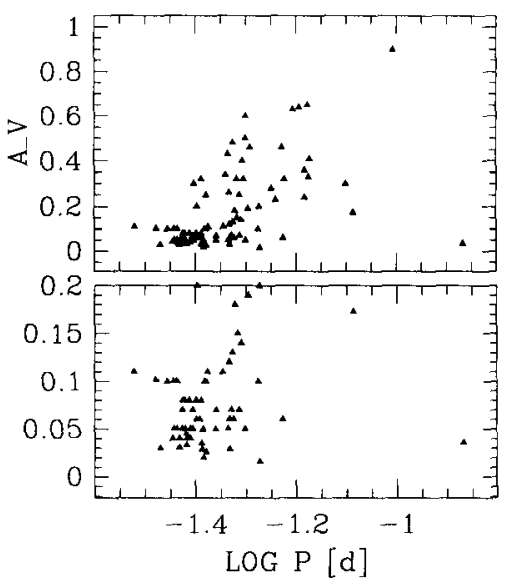

Figure 2. Full amplitude versus period for SX Phe stars identified during our survey of globular clusters.

Several side projects were attempted by the OGLE-1 team. We list here those of them which have yielded some possibly interesting results for pulsating variables. Mateo et al. (1995) searched with success for RR Lyr stars belonging to the Sagittarius dwarf galaxy. They reported $V I$ photometry for 7 variables from that galaxy. $V$ band data for $226 \mathrm{RR}$ Lyr stars from the Sculptor dwarf Spheroidal galaxy were obtained by Kaluzny et al. (1995b). That galaxy may prove to be an ideal target for calibration of the luminosity-metallicity relation for RR Lyr stars. The population of stars hosted by Sculptor shows significant range of metallicities and the interstellar reddening toward the galaxy is very low. Kaluzny et al. $(1996,1997 \mathrm{a})$ identified 34 SX Phe stars in the globular cluster $\omega$ Cen. $V$ band data for 141 RR Lyr stars ( 33 newly identified) and Pop II Cepheids in the same cluster were published by Kaluzny et al. (1997b).

In 1996 the OGLE project entered its second phase known as OGLE-2 (Udalski, Szymanski, \& Kubiak 1997a). OGLE-2 results are described in this volume by Paczyński (2000).

\section{DIRECT}

The DIRECT project (http://cfa-harvard.edu/ kstanek/DIRECT/) aims to determine the distances of the M31 and M33 galaxies by using detached eclipsing binaries and Cepheids. The project is currently conducted by a group including astronomers from CfA and Warsaw. About 200 nights on the 1.2-m FLWO and 1.3-m MDM telescopes were used between September 1996 and November 1999 to search both galaxies for variables suitable for more detailed follow-up. So far, five catalogs of variables in M31 have been released (Kaluzny et al. 1998b, 1999a; Stanek et al. 1998, 1999; Mochejska et al. 1999). 410 variables (most of them new) were identified, including 206 Cepheids and 48 eclipsing binaries. Photometry of many RV Tau and LPV stars was also reported. The remaining catalogs will be released over the coming year. Some results of DIRECT concerning Cepheids are presented in this volume by Sasselov (2000). 


\section{ASAS}

ASAS (the All Sky Automated Survey; http://www . astrouw . edu.pl/ gp/asas; Pojmański 1997) is a project whose ultimate goal is low cost monitoring of the whole sky on a nightly basis down to about magnitude 15. A prototype robotic telescope consisting of a $135-\mathrm{mm}$ telephoto lens, off-the-shelf CCD camera ( $512 \times 768$ pixels), and small automated mount was set up at the Las Campanas Observatory in April 1997. It has been monitoring 24 selected fields covering about $150 \mathrm{deg}^{2}$ of the sky. Useful photometry in the $I$ band was obtained for over 45,000 stars brighter than magnitude 13. The first two months of observation revealed 126 short period variables (Pojmański 1998), of which $70 \%$ were previously unknown. The catalogue includes several newly identified RR Lyr stars and Cepheids. In 1998 the survey was extended to cover an additional $150 \mathrm{deg}^{2}$, and the updated version of the catalogue will include data for a large number of pulsating stars with periods up to 300 days (Pojmański 1999, private communication). At the end of 1999 two new robotic telescopes with $2000 \times 2000$ CCDs will be installed at Las Campanas.

Acknowledgments. JK was supported by the Polish Committee of Scientific Research through grant 2P03D003.17, and by NSF grants AST-9528096 and AST-9819787 to Bohdan Paczyński.

\section{References}

Kaluzny, J., 1997, A\&AS, 122, 1

Kaluzny, J., Krzemiński, W., \& Mazur, B. 1993, MNRAS, 264, 785

Kaluzny, J., Krzemiński, W., \& Mazur, B. 1995a, AJ, 110, 2206

Kaluzny, J., Kubiak, M., Szymański, M., Udalski, A., Krzemiński, W., \& Mateo, M. 1995b, A\&AS, 112, 407

Kaluzny, J., Kubiak, M., Szymański, M., Udalski, A., Krzeminski, W., \& Mateo, M. 1996, A\&AS, 120, 139

Kaluzny, J., Kubiak, M., Szymański, M., Udalski, A., Krzeminski, W., \& Mateo, M. 1997a, A\&AS, 122, 471

Kaluzny, J., Kubiak, M., Szymański, M., Udalski, A., Krzemiński, W., Mateo, M., \& Stanek, K. Z. 1997b, A\&AS, 125, 343

Kaluzny, J., Krzemiński, W., \& Nalezyty, M. 1997c, A\&AS, 125, 337

Kaluzny, J., Hilditch, R., Clement, C., \& Ruciński, S. M. 1998a, MNRAS, 296, 345

Kaluzny, J., Stanek, K. Z., Krockenberger, M., Sasselov, D. D., Tonry, J., \& Mateo, M.M. 1998b, AJ, 115, 1016

Kaluzny, J., Mochejska, B., Stanek, K. Z., Krockenberger, M., Sasselov, D. D., Tonry, J., \& Mateo, M. M. 1999a, AJ, 118, 346

Kaluzny, J., Olech, A., Thompson, I., Pych, W., Krzemiński, W., \& Schwarzenberg-Czerny, A. 1999b, A\&A, in press

Kaluzny, J., Thompson, I., Krzemiński, W., \& Pych, W. 1999c, A\&A, 350, 469 
Mateo, M. M., Kubiak, M., Szymański, M., Kaluzny, J., Krzemiński, W., \& Udalski, A. 1995, AJ, 110, 1141

Mazur, B., Krzemiński, W., \& Kaluzny, J. 1999, MNRAS, 306, 727

Mochejska, B., Kaluzny, J., Stanek, K. Z., Krockenberger, M., \& Sasselov, D. D. 1999, AJ, in print

Olech, A., Kaluzny, J., Thompson, I., et al. 1999a, AJ, 118, 442

Olech, A., Woźniak, P., Alard, C., Kaluzny, J., \& Thompson, I. 1999b, MNRAS, in press

Paczyński, B. 1997, in The Extragalactic Distance Scale, ed. M. Livio, M. Donahue, \& N. Panagia (Cambridge Univ. Press), 273

Paczyński, B. 2000, in these proceedings, p. 9

Pojmański, G. 1997, Acta Astron., 47, 467

Pojmański, G. 1998, Acta Astron., 48, 35

Sasselov, D. D. 2000, in these proceedings, p. 182

Stanek, K. Z., Kaluzny, J., Krockenberger, M., Sasselov, D. D., Tonry, J., \& Mateo, M. M. 1998, AJ, 115, 1894

Stanek, K. Z., Kaluzny, J., Krockenberger, M., Sasselov, D. D., Tonry, J., \& Mateo, M. M. 1999, AJ, 117, 2810

Thompson, I., Kaluzny, J., Pych, W., \& Krzemiński, W. 1999, AJ, 118, 462

Udalski, A., Szymański, M., Kaluzny, J., et al. 1993, Acta Astron., 43, 289

Udalski, A., Szymański, M., \& Kubiak, M. 1997a, Acta Astron., 47, 319

Udalski, A., Olech, A., Szymański, M., Kaluzny, J., et al. 1997b, Acta Astron., 47,1

Woźniak, P. \& Szymański, M. 1998, Acta Astron., 48, 269

\section{Discussion}

Joergen Petersen: I have a poster on the oscillation patterns of the SX Phoenicis stars in globular clusters. Studying 48 time series taken from the literature, mostly from the OGLE project, I consider six stars as safe double-mode pulsators based on several criteria, and eight are good candidates.

Sun Hong Rhie: 1. How many exposures for each detached eclipsing binary?

2. What is the photometric accuracy?

3. How well can you determine limb-darkening parameters?

Janusz Kaluzny: 1. We are collecting from about 200 up to 1000 exposures per cluster.

2. About 0.01-0.02 mag for the turnoff stars.

3. We take them from the models. 